\section{Why simians are smart}

\author{
Carel P. van Schaik and Jan A.R.A.M. \\ van Hooff
}

Machiavellian Intelligence: Social Expertise and the Evolution of Intellect in Monkeys, Apes, and Humans. Edited by Richard Byrne and Andrew Whiten. Clarendon: 1988. Pp. 413. Hbk £50, \$75; pbk $£ 25$.

IN tests, some animals solve problems that seem to require creative insight: they show intelligent behaviour. If we accept the idea, suggested by Charles Darwin, that intelligence is an adaptation, we are forced to ask ourselves "What use is intelligence to animals?". The traditional notion is that it enables them to solve ecological problems, such as remembering where to find food and making tools to obtain it. We now know that most animals in the wild do not use tools very often or very intelligently. For instance, in captivity the orang-utan is the Houdini among animals, whereas in the forest the ape's highest achievement is to snap branches to form a night nest or scratch its back with a twig.

It was not until a more cognitive approach began to eclipse behaviourism that another idea gained prominence. As was argued most forcefully by Nicholas Humphrey, animals use intelligent behaviour particularly in their dealings with their companions. This idea is attractive because the social environment, unlike objects, is reactive, thereby favouring the animals' ability to decide flexibly on the best course of action and providing the right conditions for an 'arms race' of mental capacities. Meanwhile, reports appeared suggesting that monkeys and apes actually use these abilities in their everyday social life by forging and breaking alliances, bargaining for social favours, and even trying to deceive others by using social signals out of context. In short, they seem to behave like astute politicians. This has led primatologist Frans de Waal to draw an analogy with Machiavelli's The Prince.

In Machiavellian Intelligence, over 20 authors have been brought together in order to assess the state of this fledgling field. The book is something of a hybrid between a reader and an edited volume: almost a third of the material has been published before. The editors have included the classic papers, although some modern readers may be confused rather than enlightened by the early ones. Byrne and Whiten also invited experts to review progress, present new data or take stock of the current situation. By adding a few connecting chapters of their own in which they set out the issues clearly, they have

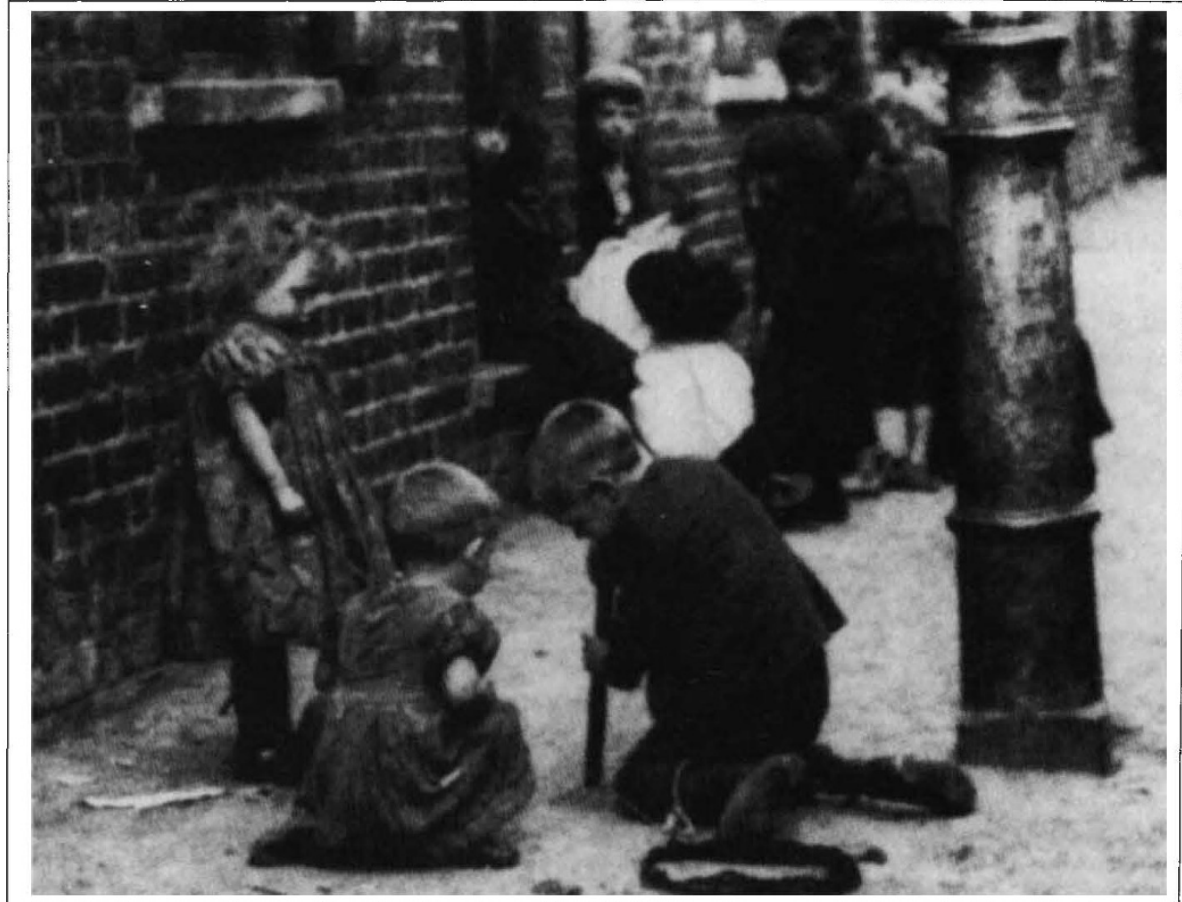

Limited expectations - East London slum life, 1899. The photograph (from the British Library) is reproduced from Atlas of Disease Distributions: Analytical Approaches to Epidemiological Data by Andrew D. Cliff and Peter Haggett. Although most of the maps are of disease patterns in the late 20th century, the atlas spans over a century. The book, to be published on 9 March by Basil Blackwell, costs $£ 75, \$ 150$.

created a highly cohesive book that will serve as an excellent introduction to social intelligence.

Several chapters are devoted to the seemingly trivial task of documenting the depth of the social knowledge of monkeys, apes and children. In practice, ingenious experiments or controlled observations were required to establish conclusively that monkeys recognize that two other individuals are relatives, or that one of them is dominant over the other. Alexander Harcourt underscores the complexity of the rules used in coalitions and thus supports the idea that intelligence evolved because of the need to make calculated decisions based on the instant evaluation of the fast-changing social scene. These contributions prepare the reader for the following chapters in which primates are shown to be "natural psychologists' who impute intentions and expectancies to others. Possession of this faculty lets them anticipate the moves of others more easily, but it also facilitates the tactical use of deception. Anyone who has watched primates is convinced that they occasionally deceive one another. Critics, however, will point to the anecdotal nature of much of the evidence, and even if they grant that deception occurs will question whether, in order to explain its most complex manifestations, we really need to assume that some apes think that they know what other apes are thinking. If it turns out that we do need to make such an assumption, then the complexity of deception would provide a yardstick for measuring intelligence.
Some chapters deal with competing ideas. The tool-use hypothesis, although inadequate when applied to primates in general, seems ideally suited to explain the rich material culture in the smartest of all primates. Ironically, the chapters concerned with human beings serve to bolster the social-intelligence hypothesis. Peter Smith shows that children reach certain developmental stages of understanding earlier with respect to people than to objects. And Thomas Wynn shows that during the phase of spectacular expansion of the human brain tools remained much the same.

Katharine Milton also takes an ecological perspective, putting emphasis on the energetic costs of maintaining large brains and thus on the factors that limit intelligence. Unfortunately, the comparative studies required to test these competing hypotheses will be difficult to carry out because we do not really know how to measure social complexity or social intelligence. The problem illustrates the point that these are early days, but at least the social-intelligence hypothesis is proving to be heuristically useful for probing the limits of social intelligence in primates. It is good scientific practice ruthlessly to apply Occam's razor, but in the case of social intelligence the simplest explanations may turn out to be those that assume higher mental abilities in the animals.

Carel P. van Schaik and Jan A.R.A.M. van Hooff are in the Laboratory of Comparative Physiology, University of Utrecht, PO Box 80086, 3508 TB Utrecht, The Netherlands. 